\title{
Cyclic Resistive Switching Effect in Plasma Electrolytically Oxidized Mesoporous $\mathrm{Pt} / \mathrm{TiO}_{2}$ Structures
}

\author{
S. Fullam, N. J. Ray, E. G. Karpov* \\ Civil \& Materials Engineering, University of Illinois at Chicago, Chicago, IL 60607, U.S.A.
}

\begin{abstract}
Understanding the resistive switching phenomenon in metal oxide semiconductors is necessary in producing reliable resistive random access memory and other variable resistance devices. An alternative technique for fabricating resistive switching elements is presented. Using plasma electrolytic oxidation, 10-11 $\mu \mathrm{m}$ thick oxide layers were galvanostatically grown on Ti substrates in a $3 \mathrm{M} \mathrm{H}_{2} \mathrm{SO}_{4}$ electrolyte. Analysis of the $\mathrm{TiO}_{2}$ layer by SEM, AFM, and XRD found the mesoporous titania surface to have a high ratio of rutile to anatase phases. The samples demonstrated pinched I-V hysteresis attributed to the resistive switching effect, when subjected to cyclic loading $( \pm 2.5,1.6,0.7 \mathrm{~V} ; 23-736 \mu \mathrm{Hz})$ at room temperature. Ratio with magnitude of 6 is reported for the resistance switching effect during $1.6 \mathrm{~V} 368 \mu \mathrm{Hz}$ loads.
\end{abstract}

\section{Introduction}

Due to concerns over the feasibility of further scaling of charge-based NAND storage [1], there is a great deal of interest in developing next-generation nonvolatile memory. Emerging technologies include STTRAM, FeRAM, MRAM, PCRAM, and resistive random access memory (RRAM or ReRAM.) RRAM shows great promise as it could reach the read/write speeds of DRAM while

\footnotetext{
* Corresponding author

Email address: ekarpov@uic.edu (E. G. Karpov)
}

Preprint submitted to Elsevier

February 19, 2015 
having a storage density and power consumption comparable to flash memory [2]. Additionally, RRAM manufacturing could be compatible with current CMOS fabrication techniques [3].

RRAM exploits the phenomena of resistive switching, where the resistivity of a material depends on its loading history. The factors affecting resistive switching behavior depend on the composition of the system; for devices based on transition metal oxides such as $\mathrm{HfO}_{2}, \mathrm{ZrO}_{2}$, and $\mathrm{TiO}_{2}$, it is generally accepted that the creation and destruction of conductive filaments of oxygen vacancies play the dominant role. $\mathrm{TiO}_{2}$ resistive switching devices have been popular candidates due to their fast switching times and long-term reliability and have been fabricated by groups using sol-gel $[4,5]$, atomic layer deposition (ALD) [6, 7] and plasma enhanced atomic layer deposition (PEALD) [8], radio frequency (RF) sputtering [9, 10], and e-beam evaporation [11, 12] methods. We report on the resistive switching characteristics of a $\mathrm{Ti} / \mathrm{TiO}_{2} / \mathrm{Pt}$ device whose mesoporous oxide layer is grown via plasma electrolytic oxidation (PEO).

The electrochemical procedure for PEO, or microarc electrolytic oxidation, is similar to traditional anodizing, but it utilizes a larger potential difference. Under sufficiently high voltages there are multiple physical phenomena that occur on the oxide/electrolyte interface; these include dissolution, dielectric breakdown, and plasma formation[13]. As the voltage reaches the dielectric breakdown of the growing oxide, spark discharges occurs which results in a porous structure with novel catalytic and corrosion properties. Although this leads to a volatile synthesis in the nano-regime, on a micro-scale the overall process leads to a uniform mesoporous structure that is easily reproducible. This process allows for the formation of a homogeneous mesoporous structure [14] that exhibits the resistive switching behavior; most notably, resistive switching occurs without the need for electroforming. Compared to ALD or PEALD, the present method is more simple: there is no reaction chamber to maintain, and only the inexpensive electrolytic solution is consumed. 


\section{Experimental Procedures}

The samples were prepared with the same two-step method outlined previously [15]. ASTM B625 Ti plates with a purity of 0.989 were cut into $36 \times 12 \times$ $0.5 \mathrm{~mm}^{3}$ substrates. Each substrate was placed $12 \mathrm{~mm}$ away from a rectangular prism graphite cathode of dimensions $153 \times 26 \times 6 \mathrm{~mm}^{3}$ and fastened into a nylon holder such that the Ti substrate was parallel to the graphite cathode. The holder was submerged in a beaker of $3 \mathrm{M}$ sulfuric acid in which the electrolyte level was more elevated than the substrate, and that beaker placed into a $4.5 \mathrm{~L}$ container of room temperature water acting as a heat sink. A constant current of $850 \mathrm{~mA}\left(93.2 \mathrm{~mA} / \mathrm{cm}^{2}\right)$ was applied for 20 minutes, reaching a maximum voltage of $155 \pm 2 \mathrm{~V}$. After 20 minutes of anodization, the current was stopped abruptly. Plasma arcing and gas emission were observed on the surface of the sample. The samples were then examined via XRD (Fig. 1a) and found to contain a high ratio of rutile to anatase phases. Next, a $34 \times 10 \mathrm{~mm}^{2}$ mask was applied, and 0.9995 pure $\mathrm{Pt}$ was deposited on the $\mathrm{TiO}_{2}$ surface at the rate of $0.02 \mathrm{~nm} / \mathrm{s}$. When the amount of Pt evaporated on the mesoporous $\mathrm{TiO}_{2}$ surface, as measured by an Inficon microbalance, reached a thickness equivalent to a 15 $\mathrm{nm}$ thick Pt film as would be the case during deposition on a flat surface, the sputtering was abruptly stopped. Two $0.5 \mathrm{~mm}$ thick wires were then attached to the surface with an SEM grade Ag paste, as shown in Fig. 1d. Finally, the surface was scanned by SEM and AFM, revealing pores with diameter approximately $200 \mathrm{~nm}$. The mesoporosity of $\mathrm{TiO}_{2}$ can be clearly seen in Fig $1 \mathrm{~b}$ and the color gradation on the AFM scan (Fig 1c) illustrates the uneven morphology of a representative area, with lighter yellow indicating an elevated region.

Each sample was subjected to a variety of loading conditions $( \pm 2.5,1.6,0.7$ V; 23-736 $\mu \mathrm{Hz}$,) and shielded from ambient light to prevent any possible photocurrent generation. One sample was also investigated under ambient, vacuum, and low oxygen environments. 


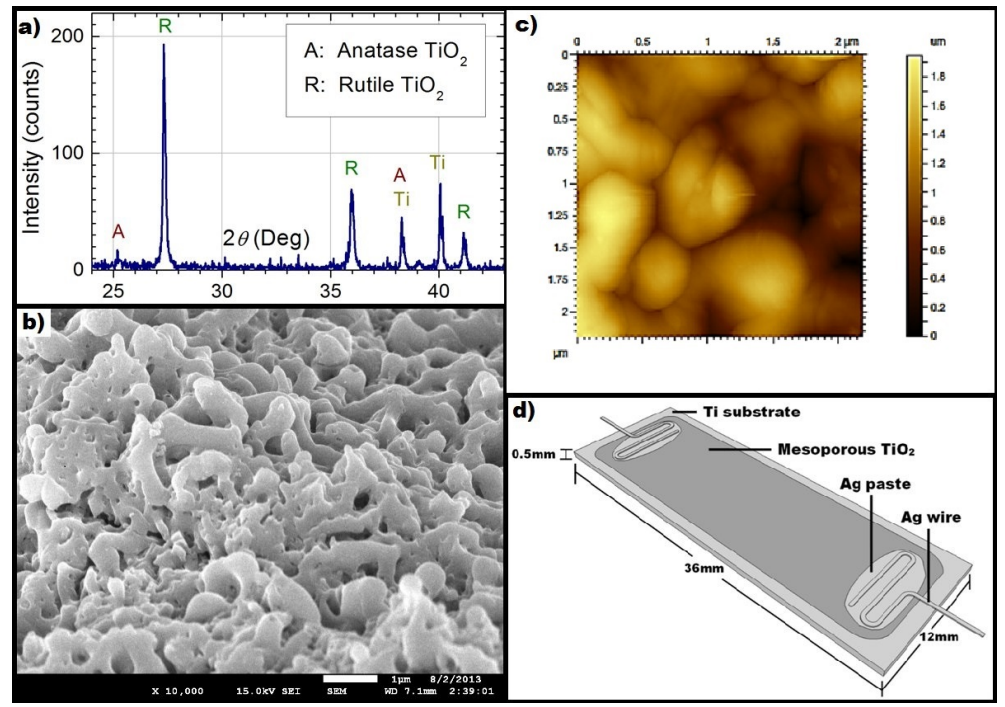

Figure 1: (a) XRD of sample surface before Pt deposition, (b) SEM micrograph of mesoporous surface, (c) AFM scan of sample area, and (d) device layout.

\section{Results and discussion}

Pinched hysteresis loops, characteristic of bipolar resistive switching, were clearly observed at $1.6 \mathrm{~V}$ and $2.5 \mathrm{~V}$ for most tested frequencies. An example of a typical I-V response for the device is shown in Fig. 2. The asymmetrical shape of the curve is due to the ohmic contact formed at the $\mathrm{Ti} / \mathrm{TiO}_{2}$ junction, and a Schottky contact at the $\mathrm{TiO}_{2} / \mathrm{Pt}$ junction[16]. As voltage is increased, current increases gradually until $+1.2 \mathrm{~V}$, then increases sharply. The region before the voltage of $1.2 \mathrm{~V}$ is classified as the high resistive state (HRS), after which further bias increase in the $1.2-1.6 \mathrm{~V}$ range changes the configuration of vacancies in the mesoporous $\mathrm{TiO}_{2}$ layer that, in turn, increases conductivity of the device. This range corresponds to the switching regime (SR) of the device leading eventually to the low resistance state (LRS) at the bias turning point, see Fig. 2. When voltage decreases, the device remains in the low resistive state corresponding to the top branch of the hysteresis loop. When a sufficiently large negative bias is applied, the device switches back to a HRS. The difference in resistivity for the HRS and LRS was larger at higher applied voltages. It is 


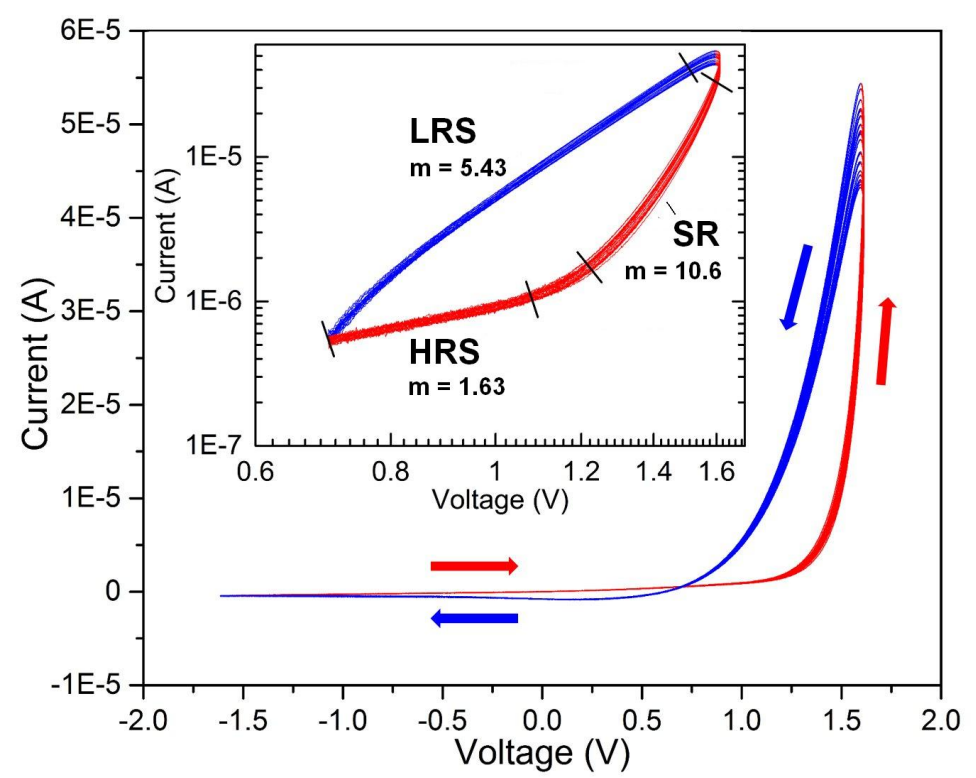

Figure 2: Pinched hysteresis of the I-V response for $\pm 1.6 \mathrm{~V} 368 \mu \mathrm{Hz}$ cyclical load. Three linear slopes $m$ are well defined on the $\log$ - $\log$ scale, $\log I=m \log V+b$, shown on the inset for the $I>0$ portion of the pinched hysteresis. These slopes correspond to the high resistance state (HRS), switching regime (SR) and low resistance state (LRS) of the device.

interesting to note that paths of the HRS and LRS intersect at around $+0.7 \mathrm{~V}$, rather than at the origin. This may be caused by a buildup of charge on the device, leading to a nanobattery effect [17].

The tested devices demonstrated well-repeatable resistive switching behavior without requiring the application of high and potentially destructive voltages that accompany electroforming steps. Smaller hysteresis loops were observed at higher frequencies, but the ratio $R_{H R S} / R_{L R S}$ increases reversibly with frequency in the 23-736 $\mu \mathrm{Hz}$ range at $1.6 \mathrm{~V}$ amplitude. This low electroforming voltage likely indicates that the PEO process creates an oxide layer with a high concentration of oxygen vacancies. We must further consider the possibility that the mesoporous oxide structure aids in oxygen evolution from the top electrode during electroforming, thus preventing localized lift-up. When one sample was loaded under vacuum conditions, it behaved as a linear resistor, $(\mathrm{R} \cong 2.5 \mathrm{M} \Omega$ ) signifying that oxygen is a factor in the resistive switching of this system. Fur- 


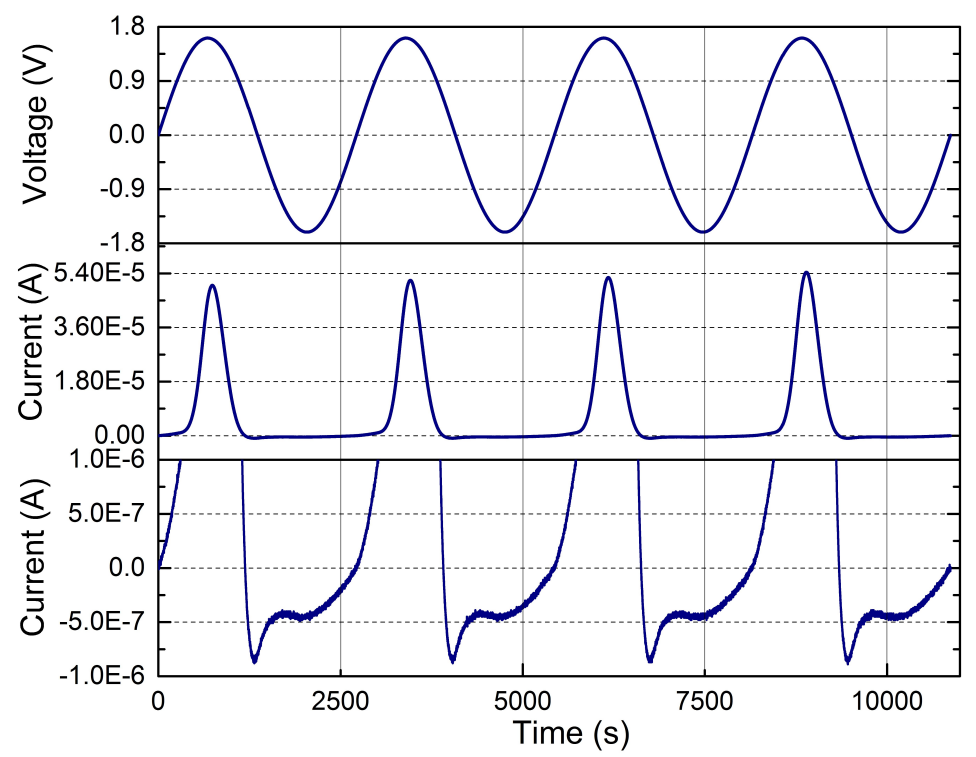

Figure 3: Applied voltage, current and zoomed-in current responses for 4 voltage cycles.

ther investigation into the relative contributions of oxygen vacancy filaments and oxygen redox reactions on resistive switching behavior is necessary. Sample endurance was studied for 25-30 cycles, shown in Fig. 2 data, and a random selection of four such cycles is illustrated in Fig. 3. The resistance of the HRS and LRS at applied potential of $1 \mathrm{~V}$ during the $\pm 1.6 \mathrm{~V} 368 \mu \mathrm{Hz}$ cyclical loading is given in Fig. 4 to depict sample stability. Over the 25 cycles displayed, both HRS and LRS exhibit relative standard error near 1\%; in the case of the HRS relative standard error was $1.02 \%$, while that of the LRS was $0.93 \%$.

Due to the fact that the PEO synthesis eliminates the need for electroforming, stability of the resistive switching process should be greatly enhanced. Further optimization of the device performance can include metal ion doping [18, 19], embedding nano-dots in the oxide layer [20, 21], adding a $\mathrm{HfO}_{2}$ layer [22], applying a compliance current [23], or decreasing the oxide layer thickness. 


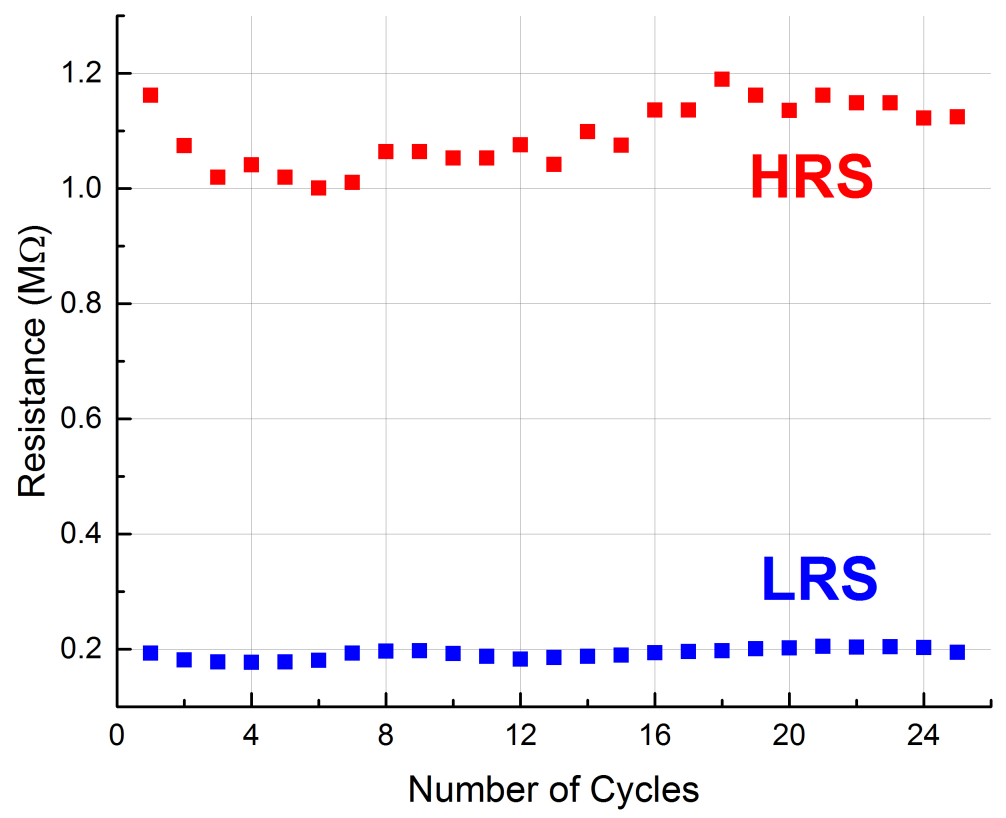

Figure 4: $\mathrm{Ti} / \mathrm{TiO}_{2} / \mathrm{Pt}$ device endurance at $368 \mu \mathrm{Hz}$.

\section{Conclusions}

Reproducible resistive switching was observed in PEO processed devices at several loading conditions. Additionally, resistive switching was observed without a high voltage electroforming step, indicating PEO yields a vacancy-rich oxide layer. The detailed microscopic mechanisms underlying switching in this system are presently unknown and warrant further investigation. While plasma electrolytic oxidation would not be compatible with existing CMOS VLSI fabrication, it provides an alternative technique for producing experimental resistive switching devices to service a wide range of applications.

\section{Acknowledgements}

This research was supported by the Center for Nanoscale Materials at Argonne National Laboratory. Use of the Center for Nanoscale Materials was 
supported by the U.S. Department of Energy, Office of Science, Office of Basic

Energy Sciences, under Contract \#DE-AC02-06CH11357. The authors thank

Dr. Song Xu, Agilent Technologies, Inc. for providing AFM scans of the device.

\section{References}

[1] R. Waser, M. Aono, Nanoionics-based resistive switching memories, Nature Materials 6 (11) (2007) 833-840. doi:10.1038/nmat2023.

[2] D. Acharyya, A. Hazra, P. Bhattacharyya, A journey towards reliability improvement of $\mathrm{TiO} 2$ based Resistive Random Access Memory: A review, Microelectronics Reliability 54 (3) (2014) 541-560. doi:http://dx.doi.org/10.1016/j.microrel.2013.11.013.

[3] C.-H. Wang, Y.-H. Tsai, K.-C. Lin, M.-F. Chang, Y.-C. King, C.-J. Lin, S.S. Sheu, Y.-S. Chen, H.-Y. Lee, F. T. Chen, M.-J. Tsai, Three-dimensional 4F2 ReRAM cell with CMOS logic compatible process, in: Electron Devices Meeting (IEDM), 2010 IEEE International, 2010, pp. 29.6.1-29.6.4. doi:10.1109/IEDM.2010.5703446.

[4] K. P. Biju, X. Liu, J. Shin, I. Kim, S. Jung, M. Siddik, J. Lee, A. Ignatiev, H. Hwang, Highly asymmetric bipolar resistive switching in solution-processed $\mathrm{Pt} / \mathrm{TiO} 2 / \mathrm{W}$ devices for cross-point application, Current Applied Physics 11 (4, Supplement) (2011) S102 - S106. doi:http://dx.doi.org/10.1016/j.cap.2011.07.018.

[5] N. Gergel-Hackett, B. Hamadani, B. Dunlap, J. Suehle, C. Richter, C. Hacker, D. Gundlach, A flexible solution-processed memristor, Electron Device Letters, IEEE 30 (7) (2009) 706-708.

[6] B. J. Choi, D. S. Jeong, S. K. Kim, C. Rohde, S. Choi, J. H. Oh, H. J. Kim, C. S. Hwang, K. Szot, R. Waser, B. Reichenberg, S. Tiedke, Resistive switching mechanism of $\mathrm{TiO} 2$ thin films grown by atomic-layer deposition, Journal of Applied Physics 98 (3) (2005) 033715. doi:http://dx.doi.org/10.1063/1.2001146. 
[7] K. M. Kim, B. J. Choi, B. W. Koo, S. Choi, D. S. Jeong, C. S. Hwang, Resistive Switching in Pt/ Al2O3/ TiO2/ Ru Stacked Structures, Electrochemical and solid-state letters 9 (12) (2006) G343-G346.

[8] S. M. Rossnagel, A. Sherman, F. Turner, Plasma-enhanced atomic layer deposition of Ta and Ti for interconnect diffusion barriers, Journal of Vacuum Science and Technology B 18 (4) (2000) 2016-2020. doi:10.1116/1.1305809.

[9] Y. H. Do, J. S. Kwak, Y. C. Bae, K. Jung, H. Im, J. P. Hong, Hysteretic bipolar resistive switching characteristics in $\mathrm{TiO} 2 / \mathrm{TiO} 2-\mathrm{x}$ multilayer homojunctions, Applied Physics Letters 95 (9) (2009) 093507. doi:http://dx.doi.org/10.1063/1.3224179.

[10] W.-G. Kim, S.-W. Rhee, Effect of post annealing on the resistive switching of TiO2 thin film, Microelectronic Engineering 86 (11) (2009) 2153-2156. doi:http://dx.doi.org/10.1016/j.mee.2009.02.028.

[11] V. Kannan, J. K. Rhee, A solution processed nonvolatile resistive memory device with $\mathrm{Ti} / \mathrm{CdSe}$ quantum dot/Ti-TiOx/CdSe quantum dot/indium tin-oxide structure, Journal of Applied Physics 110 (7) (2011) 074505-1074505-4. doi:http://dx.doi.org/10.1063/1.3644973.

[12] J.-J. Huang, C.-W. Kuo, W.-C. Chang, T.-H. Hou, Transition of stable rectification to resistive-switching in $\mathrm{Ti} / \mathrm{TiO} 2 / \mathrm{Pt}$ oxide diode, Applied Physics Letters 96 (26) (2010) 262901. doi:http://dx.doi.org/10.1063/1.3457866.

[13] M. E. Achhab, A. Erbe, G. Koschek, R. Hamouich, K. Schierbaum, A microstructural study of the structure of plasma electrolytically oxidized titanium foils, Applied Physics A 116 (4) (2014) 2039-2044. doi:10.1007/s00339-014-8392-5.

[14] A. Yerokhin, A. Leyland, A. Matthews, Kinetics aspects of aluminium titanate layer formation on titanium alloys by plasma electrolytic oxidation, Applied Surface Science 200 (1-4) (2002) 172-184. doi:10.1016/S01694332(02)00848-6. 
[15] E. G. Karpov, M. A. Hashemian, S. K. Dasari, Chemistry-Driven Signal Transduction in a Mesoporous Pt/TiO2 System, The Journal of Physical Chemistry C 117 (30) (2013) 15632-15638. doi:10.1021/jp402698f.

[16] W. Y. Park, G. H. Kim, J. Y. Seok, K. M. Kim, S. J. Song, M. H. Lee, C. S. Hwang, A pt/tio 2 /ti schottky-type selection diode for alleviating the sneak current in resistance switching memory arrays, Nanotechnology 21 (19) (2010) 195201.

URL http://stacks.iop.org/0957-4484/21/i=19/a=195201

[17] I. Valov, E. Linn, S. Tappertzhofen, S. Schmelzer, J. van den Hurk, F. Lentz, R. Waser, Nanobatteries in redox-based resistive switches require extension of memristor theory, Nature Communications 4 (2013) 1771. doi:10.1038/ncomms2784.

URL http://dx.doi.org/10.1038/ncomms2784

[18] W. Lian, S. Long, H. L, Q. Liu, Y. Li, S. Zhang, Y. Wang, Z. Huo, Y. Dai, J. Chen, M. Liu, Approaches for improving the performance of filamenttype resistive switching memory, Chinese Science Bulletin 56 (4-5) (2011) 461-464. doi:10.1007/s11434-010-4255-4.

[19] K. A. Bogle, M. N. Bachhav, M. S. Deo, N. Valanoor, S. B. Ogale, Enhanced nonvolatile resistive switching in dilutely cobalt doped TiO2, Applied Physics Letters 95 (20) (2009) 1-3. doi:http://dx.doi.org/10.1063/1.3263713.

[20] J. H. Yoon, K. M. Kim, M. H. Lee, S. K. Kim, G. H. Kim, S. J. Song, J. Y. Seok, C. S. Hwang, Role of Ru nano-dots embedded in TiO2 thin films for improving the resistive switching behavior, Applied Physics Letters 97 (23) (2010) 232904. doi:http://dx.doi.org/10.1063/1.3525801.

[21] J.-M. T. H.-J. C. F. C. M.-J. T. Wen-Yuan Chang Kai-Jung Cheng, T.-B. $\mathrm{Wu}$, Improvement of resistive switching characteristics in $\mathrm{TiO} 2$ thin films with embedded Pt nanocrystals, Applied Physics Letters 95 (4) (2009) 042104. doi:http://dx.doi.org/10.1063/1.3193656. 
[22] S. K. Kim, K. M. Kim, D. S. Jeong, W. Jeon, K. J. Yoon, C. S. Hwang, Titanium dioxide thin films for next-generation memory devices, Journal of Materials Research 28 (03) (2013) 313-325. doi:10.1557/jmr.2012.231.

[23] C. Rohde, B. J. Choi, D. S. Jeong, S. Choi, J.-S. Zhao, C. S. Hwang, Identification of a determining parameter for resistive switching of TiO2 thin films, Applied Physics Letters 86 (26) (2005) 1-3. doi:http://dx.doi.org/10.1063/1.1968416. 


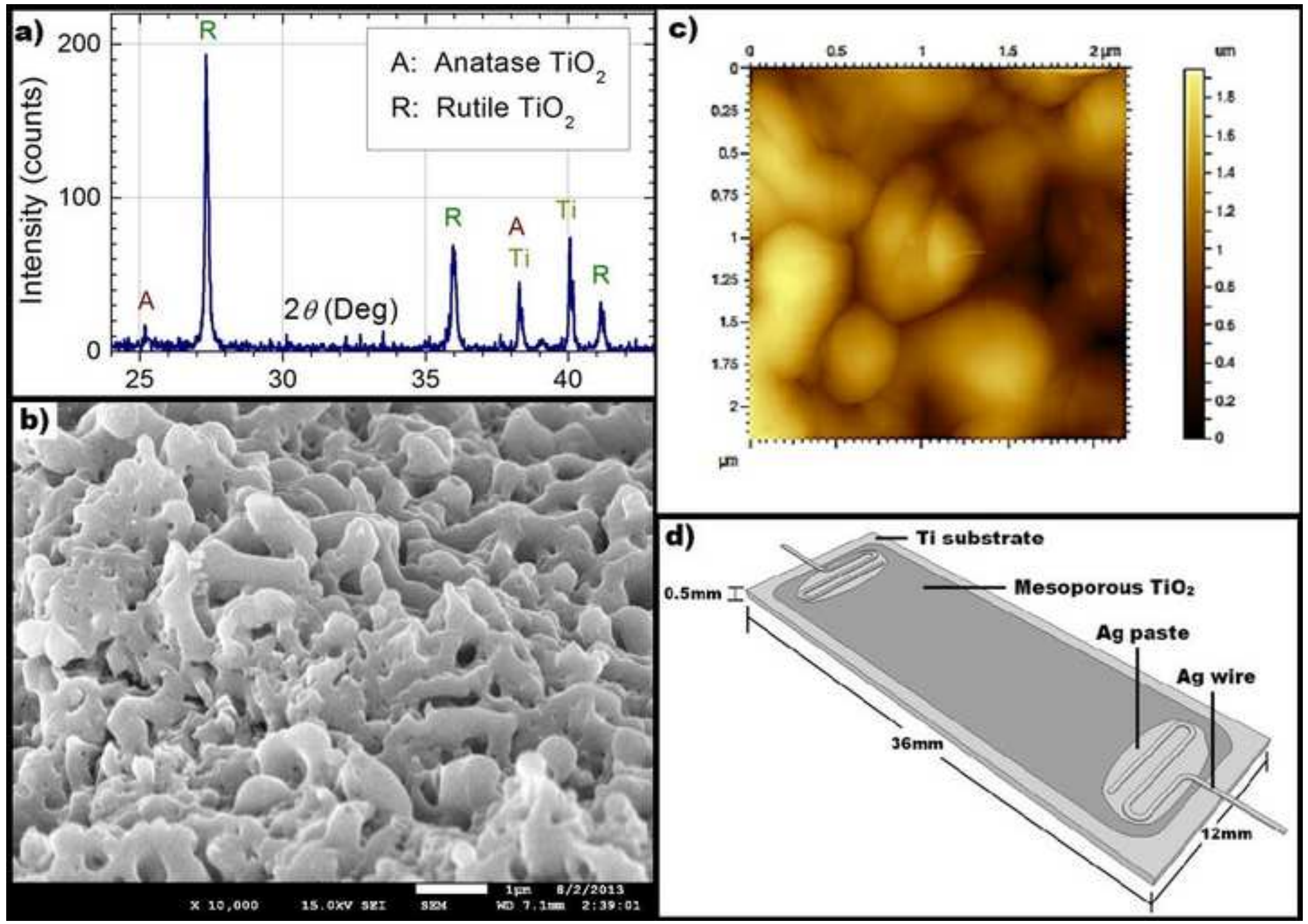




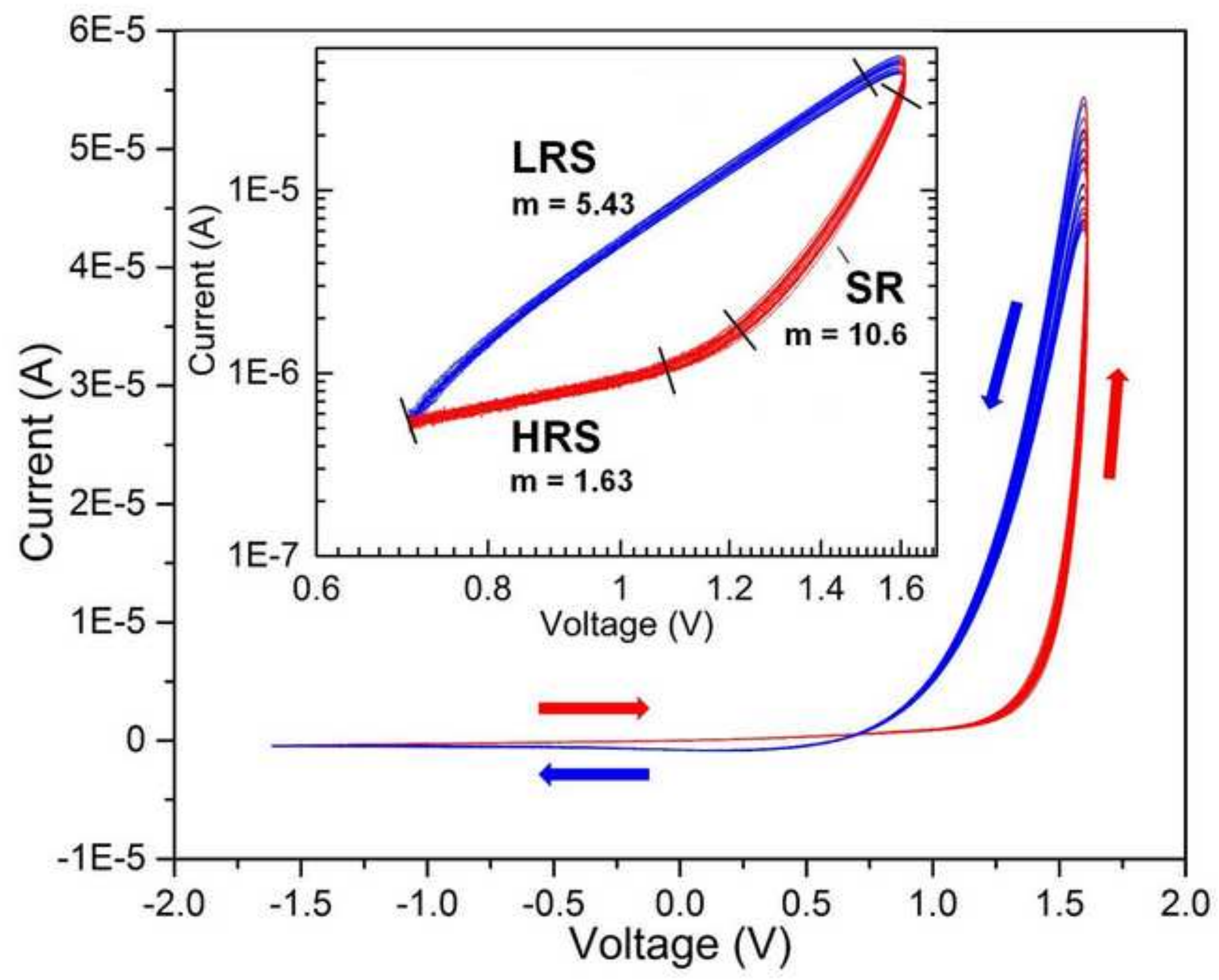




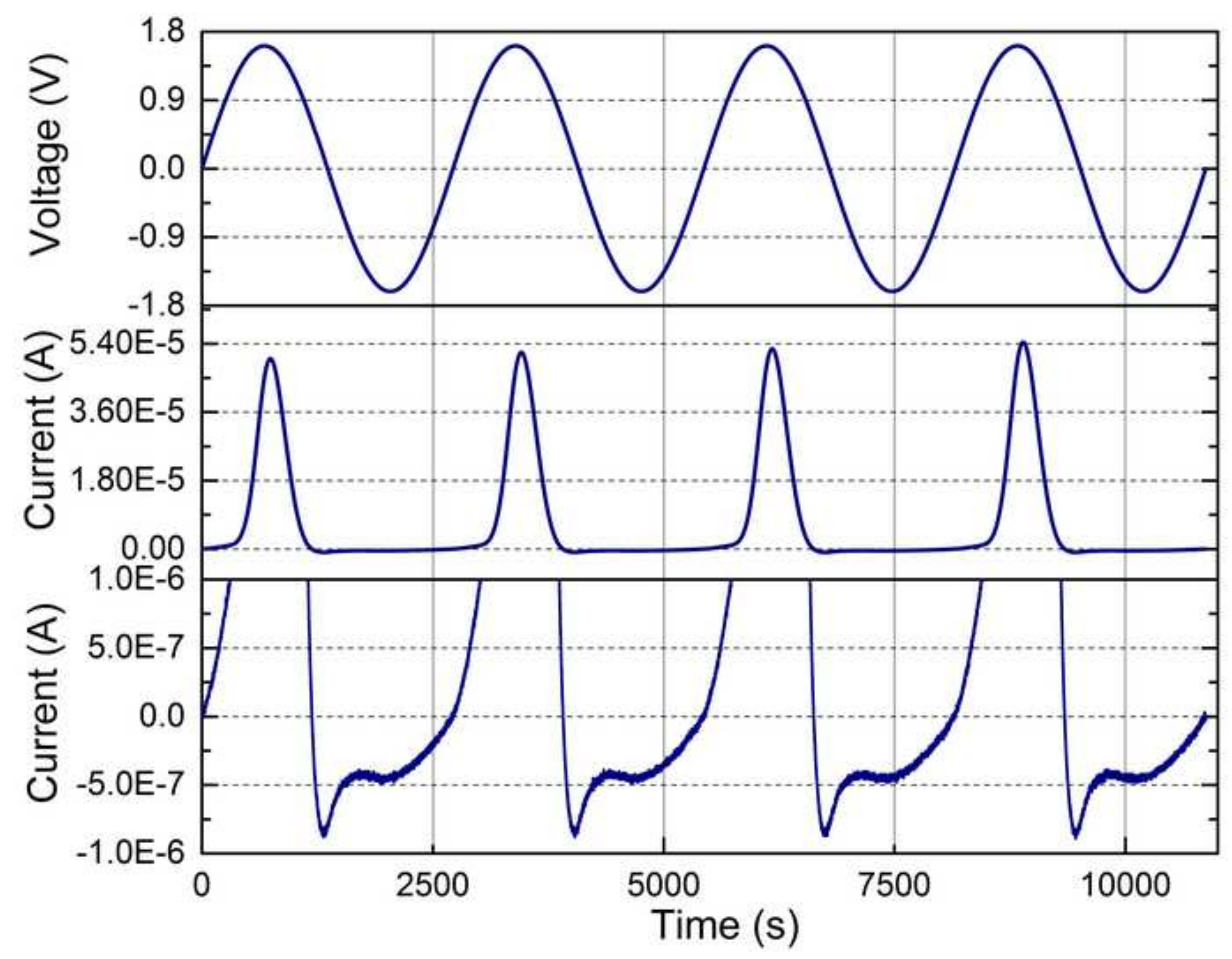




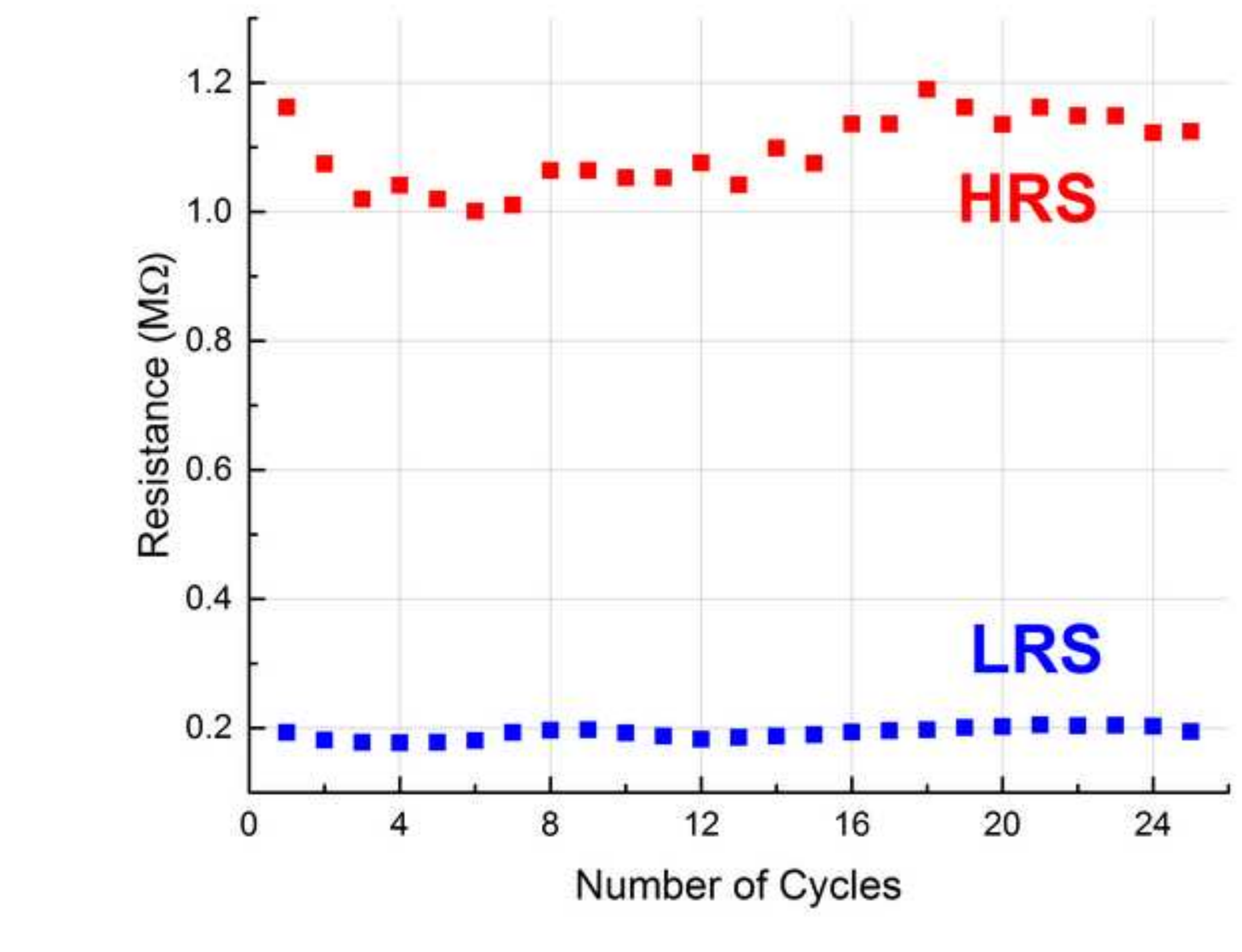

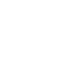

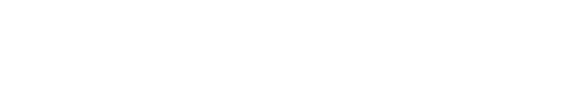

.

